\title{
A single-pieced, fully air-driven, cuff-inserted pseudo-blood pressure generator for on-site pre-screening test of non-invasive blood pressure monitor by nurses
}

\author{
Young Jun Hwang ${ }^{1}$, Gun Ho Kim², Sung Uk Yun ${ }^{3}$ and Kyoung Won Nam 1,2,3*
}

\author{
*Correspondence: \\ marmera@gmail.com \\ ${ }^{1}$ Department of Biomedical \\ Engineering, School \\ of Medicine, Pusan National \\ University, Yangsan, South \\ Korea \\ Full list of author information \\ is available at the end of the \\ article
}

\begin{abstract}
Background: It is crucial to frequently inspect the proper operation of non-invasive electronic blood pressure monitors in various sites to prevent accidents from inaccurate blood pressure measurements, especially for large-scale hospitals. However, most conventional blood pressure monitor inspection devices are not suitable for such on-site investigation purpose. In this study, we propose a new single-pieced, fully air-driven pseudo blood pressure generator that is suitable for frequent on-site prescreening tests of the blood pressure monitor by nurses.

Results: The proposed model comprises a rigid cylindrical body, two simulated brachial arteries, two air-pumps, an electronic controller, and a pressure sensor. Control algorithm based on polynomial curve fitting was implemented to generate various user-instructed systolic blood pressure and heart-rate conditions automatically. To evaluate the performance and clinical feasibility of the proposed model, various experiments were performed using ten commercial electronic blood pressure monitors. Experimental results demonstrated that the values of the Pearson coefficient between the reference pseudo-blood pressure waveforms and the actually generated pressure waveforms were $0.983,0.983$ and 0.997 at 60,70 and 80 beats $/ \mathrm{min}$, respectively $(p<0.05)$. Besides, during the experiments using ten commercial blood pressure monitors, the maximum error in average systolic blood pressure was $2.9 \mathrm{mmHg}$, the maximum standard deviation in average systolic blood pressure was $3.5 \mathrm{mmHg}$, and the maximum percentage error in average pumping rate was $3.2 \%$, respectively.
\end{abstract}

Conclusions: We expect that the proposed model can give an easy and comprehensive way for frequent on-site investigations of the blood pressure monitors by nurses, and improve the safety of patients with abnormal blood pressure, especially in most large-scale hospitals.

Keywords: Blood pressure, Investigation, Portable, Patient safety 


\section{Background}

Blood pressure (BP) is a fundamental physiological signal to understand the physical status of patients with various symptoms and diseases; therefore, most hospitals repetitively gather BP information using non-invasive electronic BP monitors whenever the patient visits [1]. When the accuracy of in-hospital BP monitors deteriorates below the clinically permissible level during long-term use, several problems can occur. For example, a misdiagnosis as abnormal BP causes unnecessary time and money expense, and risk of improper medical treatment for normal BP individuals. In the case of misdiagnosis as normal BP, individuals who need urgent attention for abnormal BP can miss the time for proper medical treatment. Therefore, it is vital to maintain the appropriate operation of in-hospital BP monitors for the safety of patients with abnormal BP $[2,3]$.

In large-scale hospitals (with hundreds or thousands beds), experts from the engineering department should periodically examine all of the BP monitors in the hospital using inspection devices; when the estimated error is beyond the permitted limit, they send it to the authorized inspection agency for fine-tuning. For this case, the best way to guarantee the proper operation of BP monitors is to inspect each device more frequently (e.g., weekly or monthly). However, in most large-scale hospitals, there are too many devices to manage (i.e., inspect, tune, and repair) compared to the number of staffs in the engineering department. For example, seven engineering staffs should manage over 4000 devices in our hospital; among them, the number of BP monitors is over one hundred. As a result, the interval of periodic BP monitor inspection is generally once per a year, which may not sufficient to guarantee the proper operation because there are many error sources in actual circumstances such as scratch in the cuff, error in embedded pressure sensor or other electronic parts, and degradation of mechanical parts due to aging.

To improve the quality of BP monitor maintenance in large-scale hospitals, it is necessary to encourage the nurses to frequently perform on-site pre-screening tests by themselves at various sites such as wards, outpatient clinics, and emergency room, and ask the engineering staffs for further inspection only when the accuracy of tested device is suspicious. However, the price of conventional inspection devices is generally high to equip plural devices at all needed sites. In addition, inspection protocol of conventional devices is somewhat complicated for untrained nurses; e.g., open the external case of BP monitor, connect an air-tube inside the inspection device to the $\mathrm{BP}$ monitor, output pre-determined pressure vibrations via the air-tube, disconnect air-tube from the BP monitor, and close the external case. To encourage such self-screening tests by nurses, it is required to develop a new inspection tool suitable for such purpose that is (1) comprehensive and straightforward for untrained nurses, (2) small, light and single-pieced to improve portability and user convenience, and (3) relatively cheap to manufacture.

In this study, we propose a new technical model suitable for on-site pre-screening tests that can generate various pseudo-BP conditions through the cuff-inserted virtual brachial arteries, and verified the feasibility of the proposed model using ten BP monitors.

\section{Results}

Figure 1a demonstrates the measurements and estimations of $P_{\mathrm{MAX}}$ when the value of PWM control register was adjusted from 0 to 240 with a step of $10\left(R^{2}=0.999\right.$ in Pearson correlation). Figure $1 \mathrm{~b}$ demonstrates the reference $P_{\text {RESP }}$ waveforms calculated by 

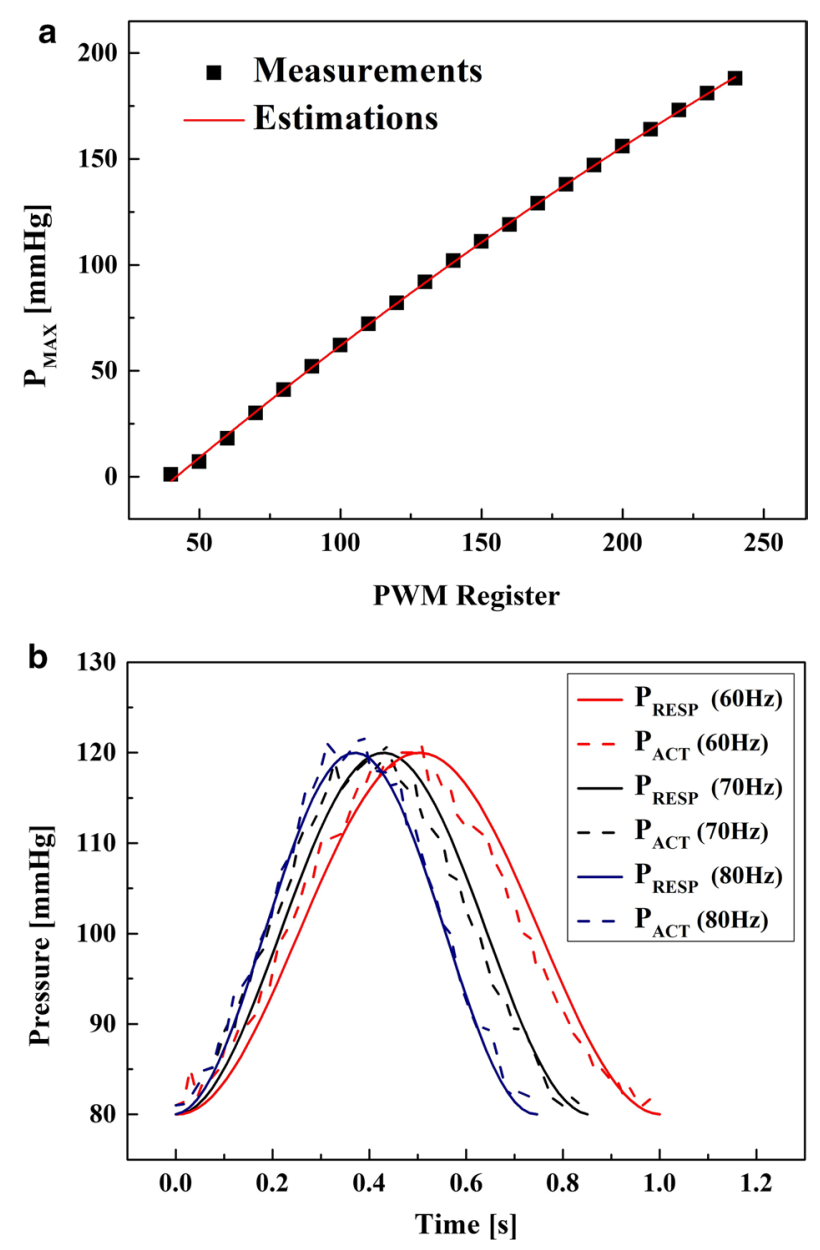

Fig. 1 Results of the performance evaluation tests. a Comparison between the measurements and estimations of $P_{\text {MAX }}$ when the PWM register was adjusted from 0 to 240. $\mathbf{b}$ Comparison between the calculations of $P_{\text {RESP }}$ (solid lines) and the measurements of pressure sensor in the implemented model (dashed lines) at 60, 70, and 80 BPM while the reference systolic BP was fixed to $120 \mathrm{mmHg}$. PWM pulse width modulation, $P_{\text {ACT }}$ measurements of pressure sensor

Eq. (2) (solid lines) and the measurements of the pressure sensor in the implemented model (dashed lines) when the $\mathrm{PR}_{\mathrm{REF}}$ was adjusted to 60, 70 and $80 \mathrm{BPM}$ while the $\mathrm{SBP}_{\mathrm{REF}}$ was fixed to $120 \mathrm{mmHg}$. In the Pearson correlation analysis, the values of the Pearson coefficient were $0.983,0.983$, and 0.997 for the curves of 60,70 and 80 BPM, respectively ( $p<0.05$ for all curves). Maximal errors between two curves were 4.9 BPM for $60 \mathrm{BPM}$, 4.7 BPM for $70 \mathrm{BPM}$, and 3.2 BPM for $80 \mathrm{BPM}$, respectively, and minimal errors between two curves were 0.1 BPM for 60 BPM, 0.0 BPM for 70 BPM, and 0.0 BPM for $80 \mathrm{BPM}$, respectively. Average errors between two curves were $2.3 \mathrm{BPM}$ for $60 \mathrm{BPM}$, 2.3 BPM for $70 \mathrm{BPM}$, and 1.2 BPM for $80 \mathrm{BPM}$, respectively.

Table 1 shows the results of $\mathrm{C} 1, \mathrm{C} 2$ and $\mathrm{C} 3$ tests. From the recorded pressure waveforms, the points around each local maximum whose value is the largest were determined as the systolic BP points, and the time intervals between two adjacent systolic $\mathrm{BP}$ points were used to calculate the actual real-time PR. In $\mathrm{C} 1$ test, average errors between the reference and the measured SBP/PR were 1.2/0.7, 1.8/0.2, 1.7/1.6, 1.0/1.1, 


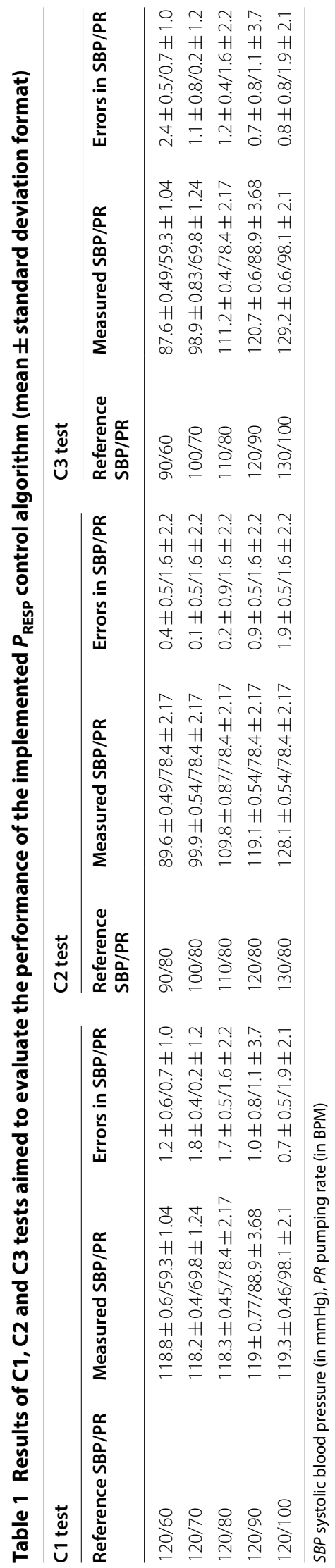


Table 2 Results of the usability test of the implemented model using ten BP monitors (mean \pm standard deviation format)

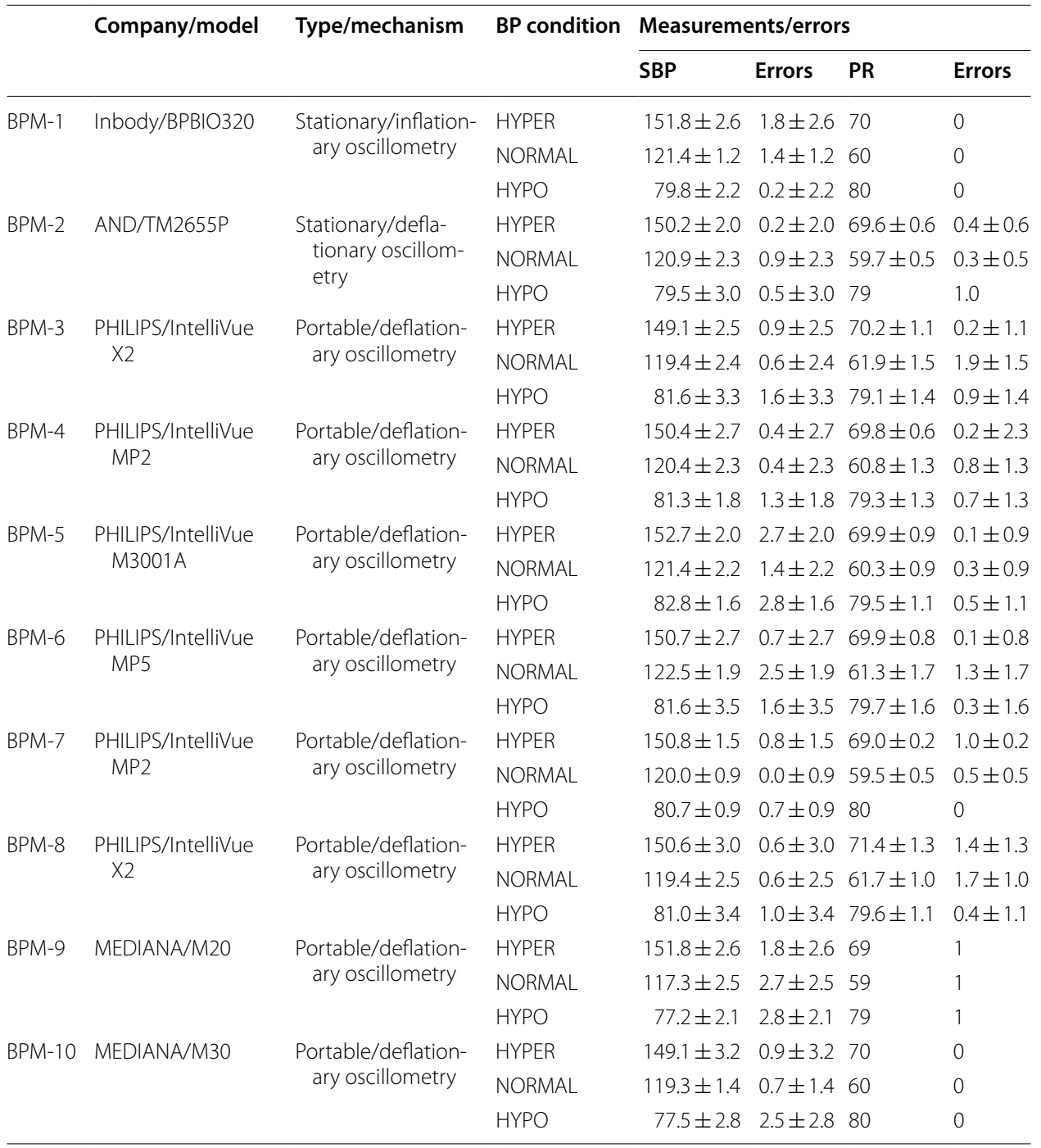

SBP systolic blood pressure (in mmHg), PR pumping rate (in BPM), HYPER hypertension, NORMAL normal, HYPO hypotension

Table 3 The measurements and errors before and after the intentional performance deterioration of the selected BP monitor (BPM-2) using the implemented model (mean \pm standard deviation format)

\begin{tabular}{llllll}
\hline & \multicolumn{1}{l}{ Before } & & & After & \\
\cline { 2 - 3 } \cline { 6 - 6 } & SBP/PR & Errors in SBP/PR & & SBP/PR & Errors in SBP/PR \\
\hline HYPER & $150.2 \pm 2.0 / 69.6 \pm 0.6$ & $0.2 \pm 2.0 / 0.4 \pm 0.6$ & & $164.9 \pm 1.3 / 69.1 \pm 0.2$ & $14.9 \pm 1.3 / 0.9 \pm 0.2$ \\
NORMAL & $120.9 \pm 2.3 / 59.6 \pm 0.5$ & $0.9 \pm 2.3 / 0.3 \pm 0.5$ & & $135.2 \pm 1.3 / 59.8 \pm 0.4$ & $15.2 \pm 1.3 / 0.2 \pm 0.4$ \\
HYPO & $79.5 \pm 3.0 / 79$ & $0.5 \pm 3.0 / 1.0$ & & $95.6 \pm 1.9 / 79.2 \pm 0.4$ & $15.6 \pm 1.9 / 0.8 \pm 0.4$ \\
\hline
\end{tabular}

SBP systolic blood pressure (in $\mathrm{mmHg}$ ), PR pumping rate (in BPM), HYPER hypertension, NORMAL normal, HYPO hypotension

and $0.7 / 1.9 \mathrm{mmHg} / \mathrm{BPM}$ when the $\mathrm{PR}_{\mathrm{REF}}$ was $60,70,80,90$ and $100 \mathrm{BPM}$, respectively. In $\mathrm{C} 2$ test, average errors between the reference and the measured SBP/PR were 0.4/1.6, 0.1/1.6, 0.2/1.6, 0.9/1.6, and 1.9/1.6 $\mathrm{mmHg} / \mathrm{BPM}$ when the $\mathrm{SBP}_{\mathrm{REF}}$ was 90, 100, 110, 120 
and $130 \mathrm{mmHg}$, respectively. In C3 test, average errors between the reference and the measured SBP/PR were 2.4/0.7, 1.1/0.2, 1.2/1.6, 0.7/1.1, and 0.8/1.9 $\mathrm{mmHg} / \mathrm{BPM}$ when the reference values of $\{\mathrm{SBP}, \mathrm{PR}\}$ varied as $\{90,60\},\{100,70\},\{110,80\},\{120,90\}$, and $\{130,100\}$, respectively.

Table 2 shows the results of the usability test using ten BP monitors. Maximal average errors between the reference and the measured systolic BPs in three test conditions (in absolute value) were 1.8, 0.9, 1.5, 1.3, 2.8, 2.5, 0.8, 1.0, 2.9, and $0.9 \mathrm{mmHg}$ at BPM$1, \mathrm{BPM}-2, \ldots$, and BPM-10, respectively. Maximal average errors between the reference and the measured PRs in three test conditions (in absolute value) were 0.0, 1.0, 1.9, 0.8, $0.5,1.3,1.0,1.7,1.0$, and $0.0 \mathrm{mmHg}$ at BPM-1, BPM-2, ..., and BPM-10, respectively. Maximal percentage errors between the reference and the measured PRs in three test conditions were $0.0,0.7,3.2,1.3,0.6,2.2,1.4,2.8,1.7$ and $0.0 \%$ at BPM-1, BPM-2, ..., and BPM-10, respectively.

Table 3 shows the measurements before and after the intentional performance deterioration (abnormal elevation of the cuff pressure measurement) of the selected BP monitor (BPM-2) using the proposed model. Before deterioration, average errors between the reference and the measured SBP/PR were $0.2 / 0.5,0.9 / 0.4$, and $0.5 / 1.0 \mathrm{mmHg} / \mathrm{BPM}$ when the test condition was HYPER, NORMAL, and HYPO, respectively. On the contrary, after intentional performance deterioration, those were $14.9 / 0.9,15.2 / 0.2$, and $15.6 / 0.8 \mathrm{mmHg} / \mathrm{BPM}$. In the independent $\mathrm{t}$-test, there were statistically significant differences in the systolic BP between two groups in all test conditions $(p<0.05)$; in contrast, there was no significant difference in the PR between two groups in HYPER and NORMAL conditions $(p>0.05)$. In HYPO condition, there was a statistically significant difference in the PR between two groups because the value of standard deviation was always zero before performance deterioration $(p<0.05)$.

\section{Discussion}

The purpose of this study is not making a new inspection device that assesses the accuracy of BP monitor; e.g., BP PUMP2 (Fluke biomedical, Washington, USA), AccuPulse (Clinical Dynamics, Wallingford, USA), AccuSim-BP (Datrend Systems Inc., Richmond BC, Canada), SC-5 SimCube (Pronk Technologies, Inc., Sun Valley, USA), and MS200 (Contec Medical Systems Co., Ltd., Qinhuangdao, China) [4]. For this purpose, it is mandatory to use an inspection device that passed technical guidelines of regulatory communities and well-designed clinical verifications, which is far from our current technical model $[5,6]$. Our target was just to implement a technical model that is suitable for quick and simple on-site pre-screening test to detect error-suspicious devices among plural in-hospital BP monitors before time-consuming official inspection in the engineering department. This kind of device may not so beneficial for consumer-selling vendors and small-scale hospitals; on the contrary, it can be useful for most large-scale hospitals that equip plural (tens or hundreds) in-hospital BP monitors at various sites but weekly or monthly inspection of each device is not possible. For these hospitals, for example, more improved management protocol can be possible using the proposed technical model as follows. First, the pseudo-BP generator is equipped in sites (e.g., wards, outpatient clinics, and emergency room) where the BP monitor is utilized. Second, nurses perform onsite pre-screening test by themselves using the equipped pseudo-BP generator weekly or 
monthly. Third, only when one or more BP monitors are suspicious during the on-site tests-e.g., the pseudo-BP generator is set to $100 \mathrm{mmHg}$ SBP but the measurements of BP monitor are consistently over $110 \mathrm{mmHg}$ or under $90 \mathrm{mmHg}-$, the nurse calls the engineering department to request further inspection.

Compared to conventional inspection devices, the proposed technical model has several advantages as follows. First, the evaluation protocol is similar to the normal BP measurement; so, nurses can easily understand how to use it. Second, it is suitable for frequent on-site inspections because it is small, light, quick, single-pieced, and nonhydraulic. Third, its manufacturing cost is relatively low; thus making it possible to equip at various sites simultaneously with less financial burden. Besides, as shown in Table 2, the proposed model demonstrated an almost even evaluation performance for BP monitors with different models and vendors. This versatility is especially important for largescale hospitals that furnish plural BP monitors with various models and vendors.

There have been a few reports that proposed cuff-insertion type BP monitor inspection devices. For example, Yong and Geddes proposed a surrogate arm that requires inserting a plastic cylindrical chamber that contains air/water mixture into the cuff [7]. However, for this device to inspect the BP monitor, additional components, such as an external water-bath, a water-filled balloon, and an air source, should be attached to the cuffinserted chamber, which makes the overall system large, heavy, and bulky. In addition, water supply and water discharge are necessary before and after the inspection; therefore, it is not suitable for quick and convenient on-site inspections. In addition, Kim et al. proposed an arm-type BP simulator that utilizes pneumatic pressure, not hydraulic pressure [8]. However, they used a bellows, servo disk motor, and screw piston for pneumatic pressure generation; therefore, it is difficult to manufacture a small and light single-pieced device, and as a result, it is not suitable for on-site inspections, too. Compared to these reports, our model does not require any large, heavy and bulky hydraulic components and can be manufactured as totally single-pieced, which improves examiner convenience.

Under the general regulatory guidelines for BP monitor, clinically permissible ranges of measurement error are (1) maximal average error in BP measurement $\leq 5 \mathrm{mmHg}$, (2) maximal standard deviation in $\mathrm{BP}$ measurement $\leq 8 \mathrm{mmHg}$, and (3) maximal average error in PR measurement $\leq 5 \%$, respectively [9-11]. As shown in Table 2, the maximal error in average SBP measurement was $2.9 \mathrm{mmHg}$ (HYPO in BPM-9), the maximal standard deviation in average SBP measurement was $3.5 \mathrm{mmHg}$ (HYPO in BPM-6), and the maximal percentage error in average PR measurement was 3.2\% (NORMAL in BPM3 ); that is, all of the three parameters satisfied the requirements of guidelines. Although more detailed verification processes, such as in vitro test, animal experiments, and clinical trials, are required in future studies, the current experimental results might show the potential of the proposed model as a BP monitor inspection device.

The limitations of the current study are as follows. First, as described above, the main purpose of the study was to make a single-pieced, fully air-driven, cuff-inserted technical model that can be used only for easy and simple on-site pre-screening purpose to detect error-suspicious devices before time-consuming official inspection. Therefore, we used a simple sinusoidal wave-profile as a reference to simplify the implementation, and fixed the difference between the $\mathrm{SBP}_{\mathrm{REF}}$ and $\mathrm{DBP}_{\mathrm{REF}}$ as $40 \mathrm{mmHg}$ based on the assumption that when the accuracy of an embedded pressure sensor in the BP monitor deteriorates, 
both of the SBP and DBP measurements will become inaccurate simultaneously. Second, the current model cannot be applied to assess the accuracy of BP monitors directly because the operating mechanism of the proposed model is far from a standard approach of the conventional BP monitor inspection devices, and as a result, it should be verified through more well-designed validation processes in future studies. Third, there have been two main approaches for benchtop assessment of BP monitors-i.e., limb simulators and waveform generators-and it is generally known that the latter is more successful than the former $[12,13]$. Although the current model showed reasonable performance during the experiments, details of the current model need to be more optimized for reliable and reproducible measurements. For example, (1) physical properties of the air-tubes and elastic meshes should be more matched to those of actual brachial artery and skin, and (2) the pseudo-BP waveform of the model should be more matched to that of actual limb.

\section{Conclusions}

In this study, we proposed a single-pieced, fully air-driven, cuff-inserted technical model for easy, simple, and quick on-site BP monitor pre-screening purpose before official inspection, and evaluated the performance and versatility of a proposed model using ten $\mathrm{BP}$ monitors. We expect that the proposed model can give a technical option to improve the quality of BP monitor management and the safety of patients with abnormal BP especially for large-scale hospitals.

\section{Methods}

Implementation of a single-pieced, fully air-driven pseudo-BP generator

In standard oscillometric BP measurement, a BP monitor detects the vibration of cuff pressure due to the occlusion and re-opening of brachial artery in the cuff during the measurement, and then, calculates average, systolic and diastolic BPs and HR [14]. To simulate this circumstance, we designed a single-pieced, fully air-driven pseudo-BP generator as Fig. 2a. Two flexible air-tubes that simulate brachial artery (diameter $=2.5 \mathrm{~cm}$, length $=19 \mathrm{~cm}$ ) are arranged outside the rigid cylinder (diameter $=7 \mathrm{~cm}$, length $=22 \mathrm{~cm}$ ) with $180^{\circ}$ spacing. To fix the position of these air-tubes during BP measurements, elastic meshes (CN03; Winner Industries Co., Ltd, Shenzhen, China) are inserted between each air-tube and the rigid cylinder, and the upper side of the air-tubes is covered by the same elastic mesh that is pulled with a constant force of about $8 \mathrm{~N}$ while winding. In the rigid cylinder, an electronic controller, two air-pumps (KPM32E; Koge Micro Tech Co., New Taipei City, Taiwan), and a pneumatic pressure sensor (MPX5100GP; NXP Semiconductors N.V. Inc., Eindhoven, Netherlands) are placed to adjust the values of upper peaks (denoting systolic BP) and frequency (denoting HR) of the pseudo-BP waveform. The generated pseudo-BP is then transferred to the cuff of the BP monitor through the air-tubes inserted into the cuff. Two air pumps, two air-tubes, a pneumatic pressure sensor, and an air-hole are connected together via polymer tubes and plastic connectors to construct a single closed air-loop. During the implementation, the cylinder and connectors were manufactured using a 3D printer (Zortrax M200; Zortrax Corp., Olsztyn, Poland). For example, to test a certain BP monitor with inflationary oscillometry mechanism, the implemented pseudo-BP generator is inserted into the cuff of the BP monitor and starts operation to generate a user-defined systolic BP and pumping rate (PR) conditions. Then, the BP monitor starts to compress the cuff-inserted pseudo-BP generator as normal 

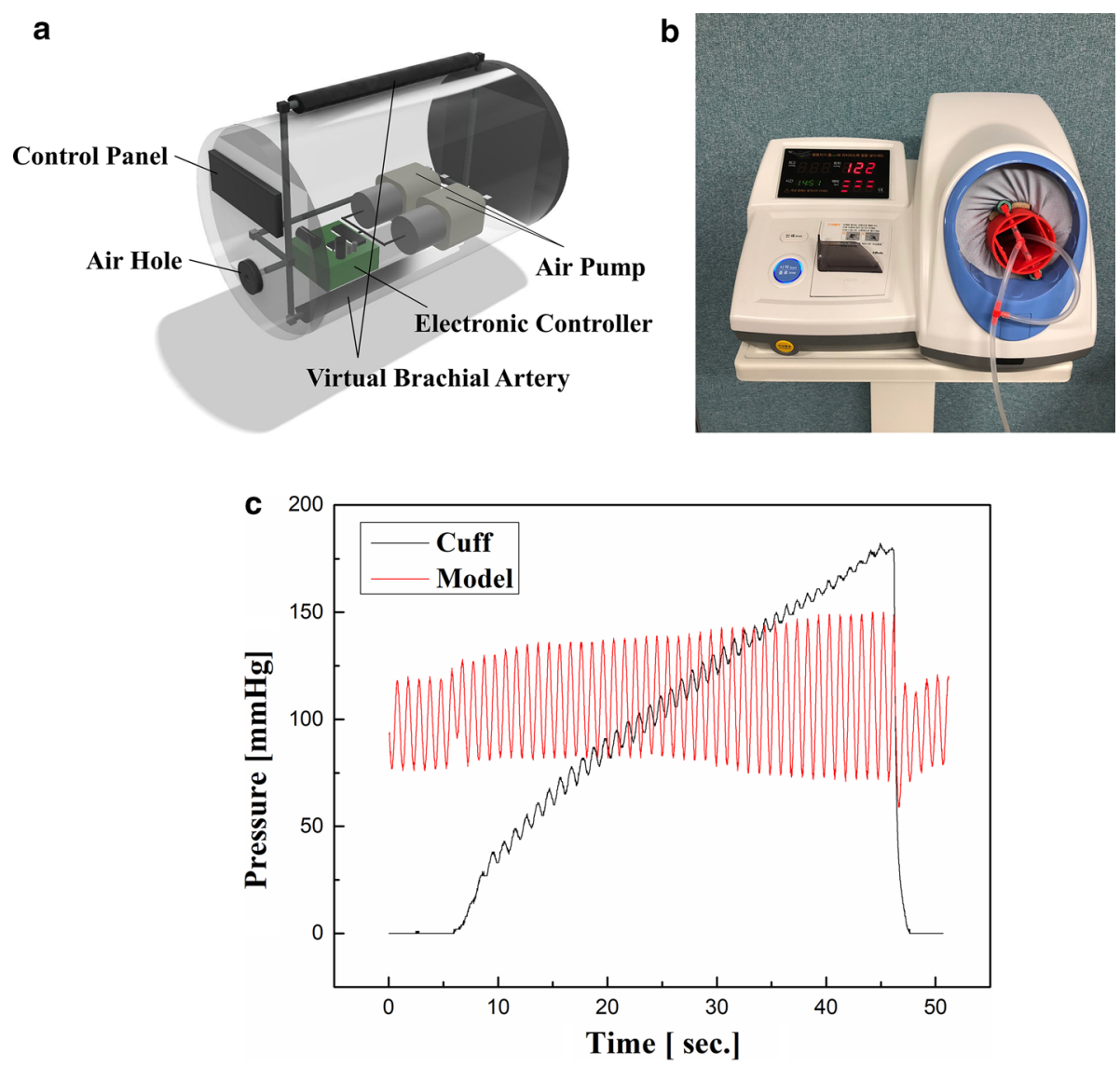

Fig. 2 Schematics of the proposed model for pseudo-BP generation. a The internal structure of the model, $\mathbf{b}$ inserting the implemented model into the cuff of BP monitor, $\mathbf{c}$ the waveforms of model-generated pressure (red line) and cuff pressure (black line) during the measurement when the model was set to $120 \mathrm{mmHg}$ systolic BP and 60 BPM PR

BP measurement situations (Fig. 2b) until the cuff pressure reaches about $300 \mathrm{mmHg}$. Then, the BP monitor measures the vibrations of cuff pressure and calculates BP and HR conditions based on its own algorithm. Figure 2c shows the waveforms of model-generated pressure and cuff pressure during the measurement when the implemented pseudo-BP generator was set to generate a pressure waveform with $120 \mathrm{mmHg}$ systolic BP and 60 beats/min (BPM) PR.

\section{Control algorithm to generate pseudo-BP waveforms through the air-tubes}

First, we set the reference waveform of the generated pseudo-BP ( $\left(\mathrm{BP}_{\mathrm{REF}}\right)$ for given $\mathrm{BP}$ and PR conditions as Eq. (1) (Fig. 3a).

$$
\mathrm{BP}_{\mathrm{REF}}(\mathrm{mmHg})=\left(\frac{\mathrm{PR}_{\mathrm{REF}}}{60}\right)\left(\frac{\mathrm{SBP}_{\mathrm{REF}}-\mathrm{DBP}_{\mathrm{REF}}}{2}\right) \sin \left(2 \pi\left(\frac{\mathrm{PR}_{\mathrm{REF}}}{60}\right) t\right)+\left(\frac{\mathrm{SBP}_{\mathrm{REF}}+\mathrm{DBP}_{\mathrm{REF}}}{2}\right),
$$

where $\mathrm{SBP}_{\mathrm{REF}}, \mathrm{DBP}_{\mathrm{REF}}$ and $\mathrm{PR}_{\mathrm{REF}}$, represent the reference values of systolic $\mathrm{BP}$, diastolic $\mathrm{BP}$ and PR by the implemented model. In our experiments, the amplitude of the pneumatic pressure in the closed air-loop reduced to $1 / N$ when the value of $P R_{R E F}$ was $\mathrm{N}$-times increased due to the decrease of pressure restoration time when $P R_{\mathrm{REF}}$ increased; to compensate for this, $\left\{\mathrm{PR}_{\mathrm{REF}} / 60\right\}$ was multiplied to the amplitude of the 


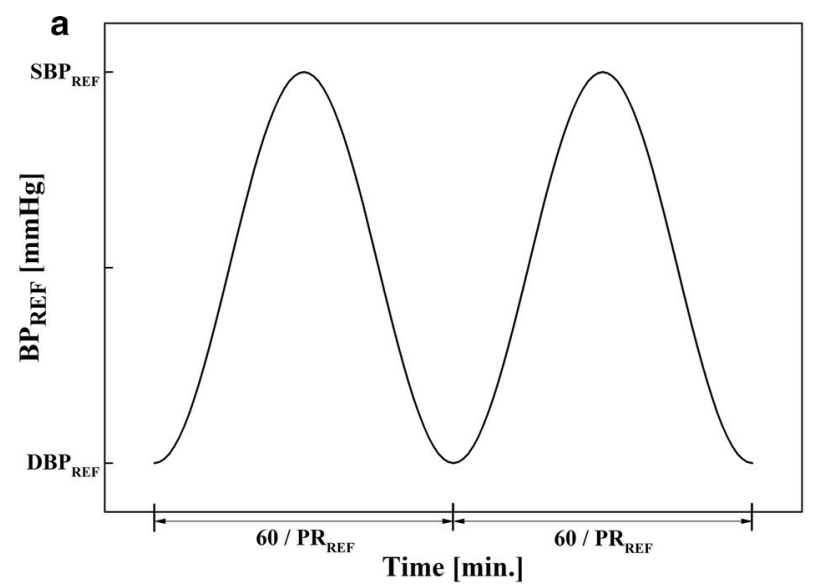

b

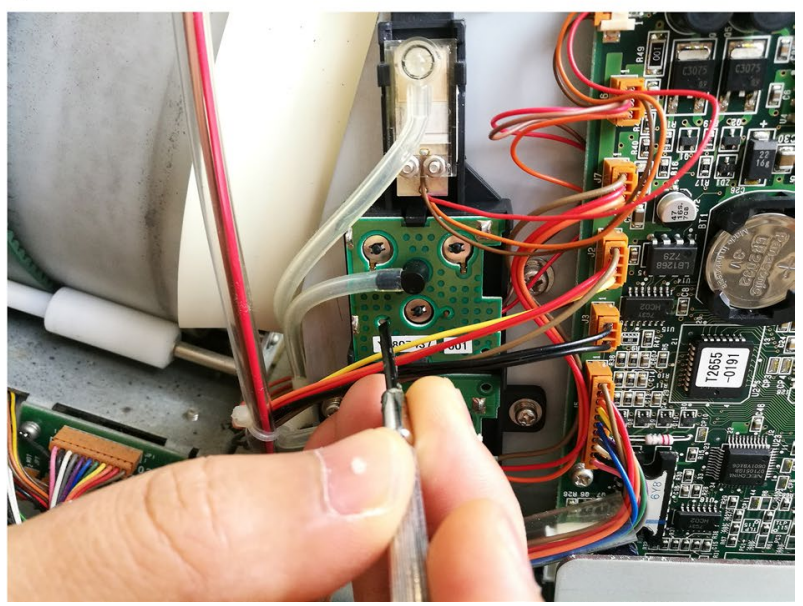

Fig. 3 Implementation of the pseudo-BP generation algorithm. a Reference waveform of the pseudo-BP given the reference values of systolic BP, diastolic BP and PR; $\mathbf{b}$ adjusting the variable resistor in BPM-2 using a screwdriver to deteriorates the accuracy of $\mathrm{BP}$ measurement

reference BP waveform in Eq. (1). Then, we further simplified Eq. (1) by assuming that the difference between the $\mathrm{SBP}_{\mathrm{REF}}$ and the $\mathrm{DBP}_{\mathrm{REF}}$ is always $40 \mathrm{mmHg}$. That is, when the examiner set the values of $\mathrm{SBP}_{\mathrm{REF}}$ and $\mathrm{PR}_{\mathrm{REF}}$, the amplitude of the $\mathrm{BP}_{\mathrm{REF}}$ waveform was automatically set to $20 \mathrm{mmHg}$ (i.e., half of the difference between the $\mathrm{SBP}_{\mathrm{REF}}$ and the $\left.\mathrm{DBP}_{\mathrm{REF}}\right)$. Then, the reference waveform moved upward to match the values of local minima with the $\left\{\mathrm{SBP}_{\mathrm{REF}}-20 \mathrm{mmHg}\right\}$, and the responsive pressure in the closed air-loop $\left(P_{\mathrm{RESP}}\right)$ that corresponds to the given $\mathrm{SBP}_{\mathrm{REF}}$ and $\mathrm{PR}_{\mathrm{REF}}$ was calculated by Eq. (2).

$$
P_{\mathrm{RESP}}=\left(\frac{\mathrm{PR} \mathrm{REF}}{3}\right) \times \sin \left(2 \pi\left(\frac{\mathrm{PR}_{\mathrm{REF}}}{60}\right) t\right)+\left(\mathrm{SBP}_{\mathrm{REF}}-20\right) .
$$

In the current study, we used an air-hole component whose hole-diameter is about $1 \mathrm{~mm}$, and empirically determined the difference between the $\mathrm{SBP}_{\mathrm{REF}}$ and the $\mathrm{DBP}_{\mathrm{REF}}$ as $40 \mathrm{mmHg}$ based on the actual $P_{\text {RESP }}$ measurements from repetitive experiments. To generate the $\mathrm{BP}_{\mathrm{REF}}$ based on Eq. (2), the electronic controller adjusts the peaks and rates of the pneumatic pressure waveform inside the closed air-loop by pulse width modulation (PWM) control. More specifically, we increased the value of 8-bit register that adjusts the duty ratio 
of PWM signal from 0 (duty ratio $=0.00 \%$ ) to 240 (duty ratio $=93.75 \%)$ with a step of 10 $($ step $=3.91 \%)$. Then, the local maxima $\left(P_{\mathrm{MAX}}\right)$ values of the generated pressure waveform at each duty ratio conditions were recorded using a pressure gauge (Heine Gamma G5; Heine Optotechnik GmbH \& Co. KG, Herrsching, Deutschland) that is also connected to the closed air-loop. Then, the relationship between the duty ratio (in \%) and the $P_{\operatorname{MAX}}$ (in $\mathrm{mmHg}$ ) was extracted by applying the polynomial curve fitting, and finally, by substituting the $P_{\mathrm{RESP}}$ in Eq. (2) for the $P_{\mathrm{MAX}}$, the relationship between the duty ratio and the $P_{\mathrm{RESP}}$ was calculated as given by Eq. (3):

$$
\text { Duty ratio }(\%)=0.000985 \times P_{\text {RESP }}^{2}+0.861 \times P_{\text {RESP }}+42.592 \text {. }
$$

\section{Evaluation of the performance of implemented model}

In this implementation, the operating ranges of $\mathrm{PR}_{\mathrm{REF}}$ and $\mathrm{SBP} \mathrm{REF}_{\mathrm{RE}}$ were restricted to 60-120 BPM and 80-150 mmHg, respectively. To evaluate the implemented $P_{\text {RESP }}$ control algorithm, the values of $P_{\text {RESP }}$ in Eq. (2) and measurements of the pressure sensor in the implemented model were compared each other in three test conditions. First, to verify the ability to adjust $\mathrm{PR}_{\mathrm{REF}}$ while maintaining the constant $\mathrm{SBP}_{\mathrm{REF}}$, (1) the value of $\mathrm{SBP}_{\mathrm{REF}}$ was fixed to $120 \mathrm{mmHg}$, and (2) the value of $\mathrm{PR}_{\mathrm{REF}}$ was adjusted from 60 to $100 \mathrm{BPM}$ with $10 \mathrm{BPM}$ step (denoted as C1 test). Second, to verify the ability to adjust $S B P_{R E F}$ while maintaining the constant $\mathrm{PR}_{\mathrm{REF}}$, (1) the value of $P R_{\mathrm{REF}}$ was fixed to $80 \mathrm{BPM}$, and (2) the value of $\mathrm{SBP}_{\mathrm{REF}}$ was adjusted from 90 to $130 \mathrm{mmHg}$ with $10 \mathrm{mmHg}$ step (denoted as $\mathrm{C} 2$ test). Third, to verify the ability to adjust both the $\mathrm{SBP}_{\mathrm{REF}}$ and $\mathrm{PR}_{\mathrm{REF}}$ simultaneously, (1) values of $\mathrm{SBP}_{\mathrm{REF}}$ and $\mathrm{PR}_{\mathrm{REF}}$ were initially set to $90 \mathrm{mmHg}$ and $60 \mathrm{BPM}$, and (2) test condition $\left\{\mathrm{SBP}_{\mathrm{REF}}(\mathrm{mmHg}), \mathrm{PR}_{\mathrm{REF}}(\mathrm{BPM})\right\}$ was adjusted to $\{90,60\},\{100,70\},\{110,80\},\{120,90\}$, and $\{130,100\}$ (denoted as $C 3$ test). Each test condition was repeated ten times during the experiments.

Next, to evaluate the clinical usability of the proposed model, ten BP monitors that were used in our hospital were randomly selected as Table 2 (BPM-1 to BPM-10; two stationary and eight portable; one inflationary and nine deflationary). Then, the implemented model was applied to each of the selected BP monitors and was operated with three representative $\mathrm{SBP} / \mathrm{PR}$ conditions [15]: $\left\{\mathrm{SBP}_{\mathrm{REF}}, \mathrm{PR}_{\mathrm{REF}}\right\}=\{150,70\}$ for hypertension (denoted as HYPER), $\{120,60\}$ for normal (denoted as NORMAL), and $\{80,80\}$ for hypotension (denoted as HYPO). All the tests were repeated 30 times during the experiments.

Next, to evaluate whether the proposed pseudo-BP generator can be used to discriminate the error-suspicious BP monitor whose measurement error is out of the permitted range, we opened the external case of an arbitrarily selected BP monitor (BPM-2) and adjusted the variable port in front of the embedded pressure sensor using a screwdriver to increase the velocity of air flow toward the sensor, which results in the elevation of the level of cuff pressure measurement (i.e., case of embedded sensor error; Fig. 3b). Then, the proposed pseudo-BP generator was applied to the modified BP monitor and the measurements at HYPER, NORMAL, and HYPO conditions were recorded 30 times each. Finally, the measurements before and after the intentional performance deterioration were compared with each other. 


\section{Authors' contributions}

YJH: designed the implemented prototype hardware and BP control algorithm, investigated the experimental data, and wrote the manuscript. GHK: performed in vitro test and investigated experimental data. SUY: designed the implemented prototype hardware and helped in vitro test. KWN: suggested the overall concept of the paper, investigated the data, and wrote the manuscript. All authors read and approved the final manuscript.

\section{Funding/acknowledgements}

This research was supported by the Korea Health Technology R\&D Project through the Korea Health Industry Development Institute (KHIDI) funded by the Ministry of Health \& Welfare, Republic of Korea (HI17C2397).

\section{Availability of data and materials}

The dataset supporting the conclusions of this article is included within the article.

\section{Ethics approval and consent to participate}

This research does not include data from human trials or animal experiments.

\section{Consent for publication}

This research does not include data from human trials.

\section{Competing interests}

The authors declare that they have no competing interests.

\section{Author details}

${ }^{1}$ Department of Biomedical Engineering, School of Medicine, Pusan National University, Yangsan, South Korea. ${ }^{2}$ Interdisciplinary Program in Biomedical Engineering, School of Medicine, Pusan National University, Yangsan, South Korea.

${ }^{3}$ Department of Biomedical Engineering, Pusan National University Yangsan Hospital, Yangsan, South Korea.

Received: 13 June 2019 Accepted: 3 October 2019

Published online: 16 October 2019

\section{References}

1. Pickering TG, Hall JE, Appel LJ, Falkner BE, Graves J, Hill MN, Jones DW, Kurtz T, Sheps SG, Roccella EJ. Recommendations for blood pressure measurement in humans and experimental animals: part 1: blood pressure measurement in humans: a statement for professionals from the Subcommittee of Professional and Public Education of the American Heart Association Council on High Blood Pressure Research. Circulation. 2005;111(5):697-716.

2. O'Brien E, Asmar R, Beilin L, Imai Y, Mallion JM, Mancia G, Mengden T, Myers M, Padfield P, Palatini P, Parati G, Pickering T, Redon J, Staessen J, Stergiou G, Verdecchia P. European Society of Hypertension recommendations for conventional, ambulatory and home blood pressure measurement. J Hypertens. 2003;21(5):821-48.

3. Speechly C, Bignell N, Turner MJ. Sphygmomanometer calibration: why, how and how often? Aust Fam Physician. 2007;36(10):834.

4. Fluke BP. Pump 2 operator's manual. https://www.manualslib.com/manual/511885/Fluke-Bp-Pump-2.html. Accessed 10 Apr 2019.

5. Stergiou GS, Alpert B, Mieke S, Wang J, O'Brien E. Validation protocols for blood pressure measuring devices in the 21st century. J Clin Hypertens. 2018;20(7):1096-9.

6. Stergiou GS, Alpert B, Mieke S, Asmar R, Atkins N, Eckert S, Frick G, Friedman B, GraßI T, Ichikawa T, loannidis JP, Lacy P, McManus R, Murray A, Myers M, Palatini P, Parati G, Quinn D, Sarkis J, Shennan A, Usuda T, Wang J, Wu CO, O'Brien E. A universal standard for the validation of blood pressure measuring devices: association for the Advancement of Medical Instrumentation/European Society of Hypertension/International Organization for Standardization (AAMI/ ESH/ISO) collaboration statement. Hypertension. 2018;71(3):368-74.

7. Yong $P$, Geddes LA. A surrogate arm for evaluating the accuracy of instruments for indirect measurement of blood pressure. Biomed Instrum Technol. 1990;24(2):130-5.

8. Kim SH, Yun SU, Cho MH, Lee SJ, Lim MH, Seo SY, Jeon GR. Development of blood pressure simulator for test of the arm-type automatic blood pressure monitor. J Sens Sci Technol. 2015;24(4):239-46.

9. IEC 80601-2-30:2009: Medical electrical equipment-Part 2-30: particular requirements for the basic safety and essential performance of automated non-invasive sphygmomanometers.

10. JIST 1115:2005: Non-invasive Automated sphygmomanometers.

11. ISO 81060-2:2013: Non-invasive sphygmomanometers_Part 2: clinical investigation of automated measurement type.

12. Mieke S. Substitution of simulators for human subjects. Blood Press Monit. 1997;2(5):251-6.

13. Balestrieri E, Rapuano S. Instruments and methods for calibration of oscillometric blood pressure measurement devices. IEEE Trans Instrum Meas. 2010;59(9):2391-404.

14. Geddes LA, Voelz M, Combs C, Reiner D, Babbs CF. Characterization of the oscillometric method for measuring indirect blood pressure. Ann Biomed Eng. 1982;10(6):271-80.

15. Guidelines Committee:2003. European Society of Hypertension-European Society of Cardiology guidelines for the management of arterial hypertension. J Hypertens. 2003;21(6):1011-53.

\section{Publisher's Note}

Springer Nature remains neutral with regard to jurisdictional claims in published maps and institutional affiliations. 